\title{
Itinerário terapêutico de brasileiros na atenção primária na tríplice fronteira
}

\section{Therapeutic itinerary of Brazilians in primary health care at triple border}

\author{
Lisete Teixeira Palma Lima', Beatriz Rosana Gonçalves de Oliveira Toso²
}

Resumo

Introdução: no Sistema Único de Saúde a atenção primária deve ser a porta de entrada aos serviços para todos os cidadãos brasileiros. Em região de fronteira, a delimitação de território para acessá-la não está submetida exatamente a definição geográfica, mas ao sentimento de pertencimento. Objetivo: conhecer o itinerário terapêutico de brasileiros, residentes no Paraguai ou na Argentina, em busca de cuidados a saúde, na atenção primária, em Foz do Iguaçu, Paraná, Brasil. Metodologia: pesquisa qualitativa, exploratória, com coleta de dados por meio de entrevistas semiestruturadas com treze usuários brasileiros não residentes no país, as quais foram realizadas em unidade básica de saúde, em 2016. Os dados foram transcritos, agrupados e analisados a partir de uma perspectiva temática. Resultados: os caminhos são percorridos com fragilidade na integralidade da atenção e na equidade das respostas do sistema de saúde em relação à pluralidade de carências desses usuários. Houve utilização similar dos subsistemas do itinerário terapêutico, entretanto o subsistema profissional foi o mais mencionado. Considerações: percebeu-se necessidade de revisão dos serviços quanto ao cumprimento dos atributos da atenção primária no cuidado de saúde aos brasileiros residentes fora do país.

Palavras-chave: Atenção primária à saúde. Acesso aos serviços de saúde. Integralidade em saúde.

\begin{abstract}
Introduction: In the Unique Health System, primary care should be the gateway to services for all Brazilian citizens. In a frontier region, the delimitation of territory to access the services is not exactly related to the geographical definition, but to their feeling of belonging. Objective: to know the therapeutic itinerary of Brazilians living either in Paraguay or Argentina searching for health assistance at primary care in Foz do Iguaçu, Paraná, Brazil. Methodology: Qualitative, exploratory research, with data collection obtained through semi-structured interviews with thirteen non-resident Brazilian users, which were performed in a basic health unit, in 2016. The data were transcribed, grouped and analyzed in a thematic perspective. Results: The pathways are covered with weakness in the attention integrality and in the equity of the health system's responses in relation to the plurality of these users' needs. There was similar use of the therapeutic itinerary subsystems, however the professional subsystem was mentioned the most. Considerations: A need to review the services was noticed, regarding the fulfillment of the primary care attributes in the health assistance for Brazilians living abroad.

Key words: Primary health care. Health services accessibility. Integrality in health.
\end{abstract}

\footnotetext{
Mestra em Biociências e Saúde, área de concentração Processo Saúde e Doença e Politicas de Saúde.

2 Doutorado em Ciências pelo programa de Enfermagem em Saúde Pública da Escola de Enfermagem de Ribeirão Preto da Universidade de São Paulo, Ribeirão Preto, São Paulo, Brasil. Professor Adjunto do curso de Enfermagem da Universidade Estadual do Oeste do Paraná, Cascavel, Paraná, Brasil. E-mail: lb.toso@gmail.com
} 


\section{Introdução}

As fronteiras são delimitadas por linhas invisíveis fora dos mapas, uma vez que estas zonas geográficas são espaços em que se criam relações que ultrapassam estes limites. ${ }^{(1)}$ Estas relações refletem o intercâmbio de mercadorias, moedas, o trânsito de pessoas em busca de serviços e ficam mais intensos quando os municípios se localizam em limites internacionais. A tríplice fronteira, que abrange Foz do Iguaçu, no Paraná, Ciudad Del Este, no Paraguai e Puerto Iguazu, na Argentina é exemplo disso. ${ }^{(2)}$

No que concerne à atenção à saúde, conforme diagnóstico local dos municípios contemplados no programa Sistema Integrado de Saúde das Fronteiras (SIS Fronteiras), do Ministério da Saúde, Foz do Iguaçu atende mensalmente 41.627 usuários estrangeiros nos seus serviços de saúde. ${ }^{(3)}$ Dentre estes, estão os brasileiros que residem nos países da fronteira e voltam ao seu país natal em busca de atenção à saúde, por meio da atenção primária.

Na concepção de atenção primária, esta deve funcionar como a porta de entrada do sistema de saúde. Utiliza tecnologias de elevada complexidade e baixa densidade, que devem resolver os problemas de saúde de maior frequência e relevância em seu território. É o contato preferencial dos usuários com os sistemas de saúde. Orienta-se pelos princípios da universalidade, acessibilidade e coordenação do cuidado, vínculo e continuidade, integralidade, responsabilização, humanização, equidade e participação social. ${ }^{(4)}$

Para atender à população usuária do Sistema Único de Saúde (SUS), na Atenção Primária a Saúde (APS), Foz do Iguaçu oferta serviços em oito Unidades Básicas de Saúde (UBS), 15 Unidades de Saúde da Família (USF), onde estão inseridas 35 equipes da Estratégia Saúde da Família (ESF) e 26 equipes de saúde bucal; distribuídas em cinco distritos sanitários. $^{(5)}$

A respeito dos caminhos que definem os diferentes modos de trilhar o sistema oficial, independente de existirem protocolos e fluxogramas bem estabelecidos pela rede assistencial, autores ${ }^{(6)}$ referem que os indivíduos desenham sua trajetória singular, por meio de escolhas emolduradas pelas suas próprias necessidades, concepções, estigmas e determinantes sociais.

O conceito de itinerário terapêutico adotado é dividido em subsistema familiar, profissional e popular. No primeiro, a doença é inicialmente identificada e a partir daí acontecem os primeiros atos e decisões em busca de tratamento. O segundo inclui a biomedicina, propostas de cura legalizadas e organizadas, com sistematização nos registros, ou seja, as ofertas dos sistemas de saúde. Por fim, o subsistema popular, que não apresenta legalidade ou organização formal e sistematizada, com oferta de especialistas de cura informais e amplo reconhecimento pela sociedade. ${ }^{(7)}$

Como os serviços citados são utilizados pelos brasileiros que residem nos outros países da fronteira com Foz do Iguaçu, que caminhos são percorridos e como acontece esse acesso, são elementos que integram o itinerário terapêutico em região de fronteira destes usuários em busca de saúde, objeto deste estudo.

Assim, tem-se por objetivo conhecer o itinerário terapêutico de brasileiros, residentes no Paraguai ou na Argentina, em busca de cuidados a saúde, na atenção primária, em Foz do Iguaçu, Paraná, Brasil.

\section{Metodologia}

Pesquisa qualitativa exploratória, ${ }^{(8)}$ cuja coleta de dados ocorreu em UBS de Foz do Iguaçu, Paraná, a qual é destinada formalmente como referência para atendimento de brasileiros que residem no Paraguai (designados como brasiguaios) e na Argentina, além de uma população local de 15.000 pessoas. Os brasileiros residentes na Argentina foram entrevistados em seu local de trabalho, uma vez que não compareceram para consulta durante o período de coleta de dados, o qual compreendeu os meses de fevereiro e março de 2016. 
Os dados foram coletados pela pesquisadora por meio de entrevistas semiestruturadas, a partir da questão norteadora: conte-me os caminhos percorridos para ser atendido até a vinda para esta Unidade de Saúde. Cada entrevista durou em média 40 minutos e os relatos foram gravados. Os critérios de inclusão foram: ser brasileiro, não ser residente em Foz do Iguaçu e residir em cidades dos países vizinhos; ter mais de 18 anos ou, se menor, estar acompanhado de responsável (os quais foram os respondentes e assinaram o termo de consentimento) e, participar voluntariamente da pesquisa. Foram excluídos os brasileiros ou estrangeiros que residem em Foz do Iguaçu, no Brasil, pois como residentes, integram os critérios da atenção básica (territorialização, cadastro familiar, vinculação à uma unidade básica de saúde) para cuidados à saúde. O número de entrevistas não foi determinado a priori e definiu-se em 13 participantes, a partir das transcrições simultâneas à realização de cada entrevista, leitura do material e verificação da qualidade do material coletado, a fim de responder ao objetivo do estudo, associado ao critério de saturação.

As falas citadas no texto foram identificadas com a letra $\mathrm{E}$, de entrevistado, seguida do número da entrevista, por exemplo, E1. A análise do material foi temática, seguindo as etapas de leituras flutuantes, em que foram sendo destacadas as unidades de sentido no texto, leituras verticais e transversais. Após os destaques das estruturas de relevância, foram agrupadas em sentidos convergentes e divergentes, para construção das unidades temáticas, ${ }^{(9)}$ as quais foram discutidas a partir da literatura pertinente, de acordo com o esquema apresentado na Figura 1.

Figura 1 - Esquema analítico.

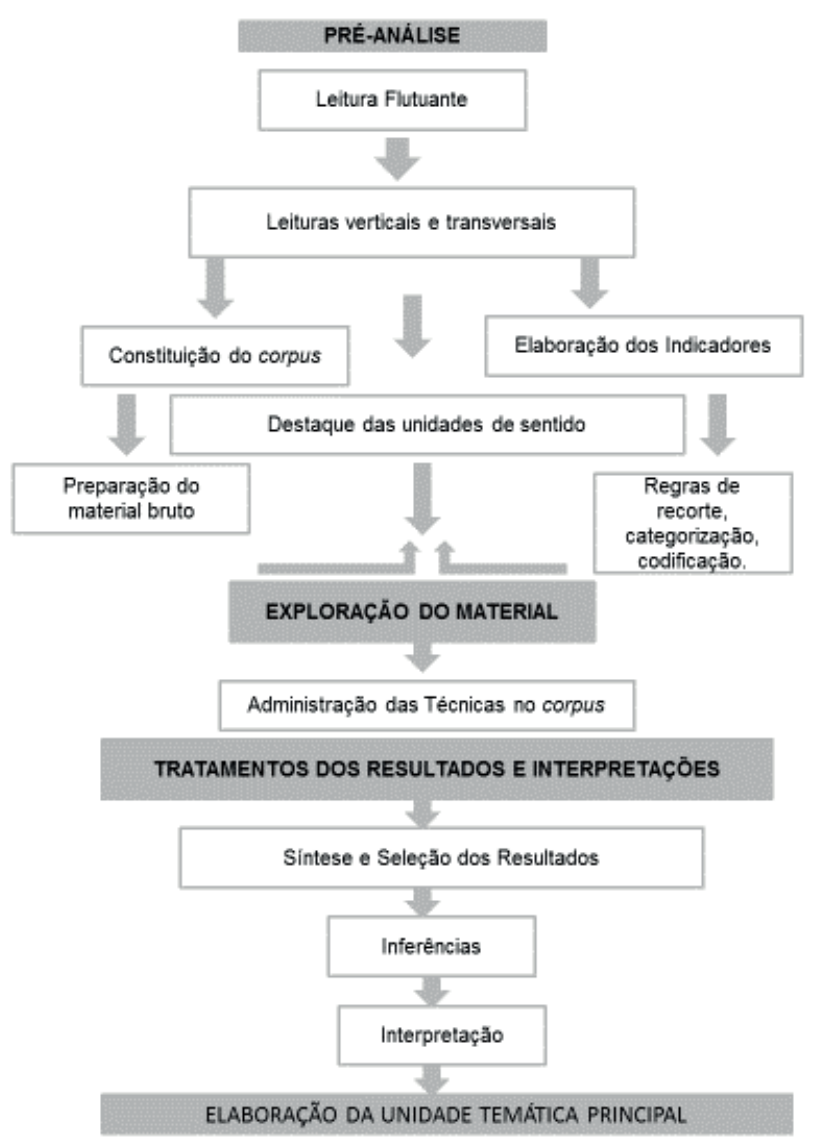

Fonte: Elaborado pelas autoras. 
Foram seguidos os preceitos éticos da resolução 466/2012-CNS e o estudo foi aprovado pelo Comitê deÉtica em Pesquisa com Seres Humanos sob Parecer $n^{\circ}$ 1.180.900 e CAAE $n^{\circ}$ 47186015.7.0000.0107.

\section{Resultados e discussão}

Inicialmente apresenta-se a caracterização dos entrevistados, cuja maioria foram do sexo feminino, brancos, com idade entre 35 e 55 anos, com ensino fundamental incompleto, casados, com um a três filhos, que trabalham sem vínculo formal, tem renda familiar até dois salários mínimos brasileiros, correspondente a 880,00 reais à época do estudo, e residem no estrangeiro entre dez e 45 anos. Como nesse estudo, artigos ${ }^{(10-11)}$ que caracterizaram a demanda de unidades de atenção primária apresentaram resultados similares para essas características.

As distâncias entre a residência destes usuários e a unidade de saúde na qual buscaram o cuidado, no Brasil, variaram de 12 a $210 \mathrm{Km}$, o que significa um trajeto com duração entre 30 minutos a 3 horas de viagem, considerando o trânsito da Ponte da Amizade (que liga Foz do Iguaçu, no Brasil, a Ciudad Del Este, no Paraguai), que faz parte do caminho obrigatório e é conhecida nacionalmente pelo intenso tráfego e congestionamento.

A unidade temática principal ora apresentada foi denominada "itinerário terapêutico de brasileiros na tríplice fronteira" e está apresentada e discutida em quatro subunidades: o público e o privado na assistência à saúde para os brasiguaios; a decisão de procurar o SUS no Brasil; o acesso aos serviços de saúde e a atenção primária à saúde como (des) coordenadora da rede de atenção.

O público e o privado na assistência à saúde para os brasiguaios

A utilização do sistema público de saúde em paralelo ao sistema privado, no caso dos brasiguaios é frequente, uma vez que buscam atendimento em clínicas privadas, para especialidades. Quando o custo se torna elevado para o usuário, este retorna ao setor público, denotando a existência de um sistema duplo, que gera o fracionamento das ações e impede o uso dos fluxos definidos, quando existentes. Essa duplicidade de utilização do sistema aparece nas falas abaixo:

[...] tenho problema de coração há 5 anos, fui no Posto São João aqui, fiz exames aqui no Brasil e depois no Paraguai, 'lá tudo pago' [...] semana passada piorei, dor forte no peito, fui pro médico e fui internado lá. 'Não tinha como pagar, vim pela emergência' [...] pensei vou pra minha terra porque ir pra Argentina, meu Deus, eu sou brasileiro, vou pra minha terra. (E1)

Isso não faz muito tempo, a gente começou a se sentir mal e veio procurar recurso, foi consultando (particular) e foi a um ponto que foi encaminhado para o SUS e fez o tratamento necessário [...] no cardiologista, fui consultado por ele [...] Pagando sempre pagando. (E10)

No caso dos brasileiros residentes na Argentina, a utilização mista entre os sistemas público e privado acontece de forma diferente do relatado pelos residentes no Paraguai, uma vez que utilizam o público, usufruindo ou da gratuidade, ou das obras sociais pagas na Argentina e, ao optarem pelo Brasil utilizam, prioritariamente, serviços privados: “[...] Tivemos vários problemas de saúde e sempre fomos à Foz do Iguaçu para resolver. Pagamos uma clínica particular aqui [...] obras sociales, mas uma coisa mais séria, dai vai para Foz [...] lá sempre paguei particular, não tem obras sociales". (E12)

$\mathrm{Na}$ busca por assistência em saúde deve-se considerar que a utilização dos serviços resulta da interação do comportamento do indivíduo que procura cuidados e da forma como é conduzido dentro do sistema de saúde. Estudo ${ }^{(12)}$ que analisou o acesso de usuários de uma Unidade Básica de 
Referência aos serviços de saúde de um município da região Metropolitana de Belo Horizonte, abordando os fatores que influenciam o acesso em unidade básica de saúde, analisou as dimensões estrutural (financiamento, cobertura), relacional (compreensão do processo saúde-doença) e operacional (organização dos serviços). Verificaram que o subfinanciamento do sistema de saúde constituiu uma dificuldade para o acesso e para a implantação da Estratégia Saúde da Família (ESF); a ausência de alinhamento entre a estrutura e os fluxos de funcionamento do sistema com impacto negativo na relação do profissional com o usuário; e lacunas como baixa cobertura da ESF, marcação de consulta, acolhimento, barreiras geográficas, problemas na referência e contrarreferência. Considera-se que todos os fatores mencionados influenciam o itinerário terapêutico do usuário no sistema de saúde, a exemplo do ocorrido nesse estudo.

Segundo a Agência Nacional de Saúde Suplementar, ${ }^{(13)} 27,1 \%$ dos brasileiros, no Paraná, tem planos de saúde. Sendo que, alguns destes planos, muitas vezes, não englobam todos os procedimentos necessários, resultando em utilização mista do convênio, do setor privado e também do SUS. O mesmo acontece com os brasileiros que residem no Paraguai com a diferença que, ao chegar à decisão de utilizar o serviço público, muitos atravessam a fronteira e o fazem no lado brasileiro ou, utilizam o serviço privado no Brasil, ou ainda ambos.

O modelo assistencial brasileiro atual, na atenção primária, tem a saúde da família em sua centralidade e promoveu ampliação do acesso e incorporação do acolhimento e humanização das práticas. No entanto, verifica-se a persistência no tratamento de patologias e dos cuidados ao corpo biológico, as dificuldades na implantação da integralidade e déficits na formação das equipes e nas condições e relações de trabalho, o que influencia no itinerário terapêutico dos indivíduos. ${ }^{(14)}$
A decisão de procurar o Sistema Único de Saúde no Brasil

Buscar a cura para suas doenças, alívio para suas dores e a retomada da vida cotidiana, são ações que podem sofrer a influência de parentes, amigos, das experiências anteriores bem-sucedidas ou não, da existência de vínculo com o profissional de saúde, entre outros aspectos. Considera-se o âmbito das relações familiares o primeiro subsistema do itinerário terapêutico. ${ }^{(15)}$ Ainda, para cada novo processo de doença e cura podem emergir itinerários diferentes. ${ }^{(7)}$

Ademais, os grupos sociais e religiosos, que podem compor o itinerário terapêutico, também foram citados nesse estudo, como se evidencia nas falas: “[...] Dra. I (se referindo à pastora da igreja que frequentava) já tinha falado que era perto do Ceasa". (E1) "[...] dai falei com algumas pessoas para vir prá cá, consultar aqui, umas irmãs da igreja". (E6)

Assim, a definição do itinerário não é circunscrita aos serviços de saúde, as opções podem variar e se multiplicar, sem conferir qualquer preponderância às soluções do setor saúde. Para não enxergar apenas parte de uma travessia, que é maior e mais complexa, é preciso compreender o itinerário não somente pelo vagar por instâncias médico-hospitalares, pois esta não é a única trajetória dos indivíduos, cujos itinerários recorrem às possibilidades oferecidas também pela religião. ${ }^{(16-17)}$

Considera-se, portanto, que a saúde é um processo social dinâmico relacionado com outros processos sociais, no qual cada indivíduo constrói e entende sua saúde e sua doença por meio da representação sociocultural em consonância com a realidade em que vive. ${ }^{(18)}$

Destarte, a influência de amigos ou pessoa fora do círculo de amigos ou familiar, mas com experiência positiva anterior no processo de cura, também pode influenciar nos motivos pela procura de determinado serviço de saúde, conforme relatos: 
[...] começamos a conversar com os outros, a gente conversava com os outros lá enquanto ela ficava internada e um cara ali me disse pra levar pro Rio Grande do Sul (reportando caminho anterior do itinerário terapêutico atual) que lá ia resolver [...] e dai fomos (pai). (E2)

[...] Nem juntando os amigos aqui (na Argentina) a gente tinha como pagar, na época 41.000 reais [...] Mas dai um brasileiro amigo nosso, foi lá e conseguiu passar tudo pro SUS, foi a salvação nossa. (E12)

Outro fator que influencia a procura pelo serviço em Foz do Iguaçu é o vínculo de confiança com o serviço ou com os profissionais de saúde, conforme se evidencia no depoimento: [...] "venho aqui porque prefiro não confiar no tratamento de lá, porque as poucas vezes que fui lá, o médico já queria me operar urgente, consultei por causa da vesícula, passei alguma dor, mas quando ele falou na operação, na cirurgia, achei melhor vir pra cá". (E8)

$\mathrm{O}$ vínculo é um fator preponderante na escolha do serviço de saúde, uma vez que amplia a eficácia das ações de saúde e favorece a participação do usuário durante a prestação do serviço. Esse espaço deve ser utilizado para a construção de sujeitos autônomos, tanto profissionais quanto pacientes, pois não há construção de vínculo sem que o usuário seja reconhecido na condição de sujeito, que fala, julga e deseja. ${ }^{(17-18)}$

Caminhando na subjetividade, o cuidado pode ser compreendido quando o sentido da ação supera o alcance de um estado de saúde desejado previamente, quando é permeado pela relação entre finalidades e meios, num diálogo horizontal entre profissional e paciente, considerando que esse último adota uma confiança quase incondicional em seu cuidador, em decorrência da fragilidade imposta pelo adoecimento. ${ }^{(19)} \mathrm{O}$ cuidado pode ser transportado, nesse estudo, como vínculo, uma vez que estes brasileiros, além da busca pelo cuidado, têm a confiança no serviço do seu país de origem.

Outro fator que levou à procura do serviço em Foz do Iguaçu foi a influência de familiares, conforme o depoimento: "[...] na verdade, foi a minha cunhada, acho que ela vinha aqui quando estava doente [...] Sim, ela que me orientou a vir aqui, eu não conhecia aqui, na verdade". (E11)

Ainda, outra possibilidade dentro dos itinerários terapêuticos, na tentativa de conseguir a cura ou restabelecimento de uma situação de saúde próxima ao esperado, são os tratamentos alternativos ao sistema oficial, conforme depoimento: "Começou com uma dor de estômago, há, acho, mais ou menos um ano atrás, dai aquela coisa, fui na farmácia, tomei remédio, lá é assim, a gente vai na farmácia se resolver, resolveu". (E7)

Sobre a busca de solução para um problema de saúde diretamente com a automedicação ou com a orientação recebida em farmácias, de modo geral, há uma tendência em construir modelos com os quais é possível explicar as escolhas que os indivíduos fazem sobre terapêutica: os modelos etiológicoterapêuticos ou de relação causa-tratamento, o modelo de decisão terapêutica, o modelo de crenças de saúde, os modelos explicativos, e as justificativas eminentemente práticas, baseadas em ações do cotidiano. A maior parte dessas abordagens sugere que o sujeito, ao identificar uma doença opta por este ou aquele recurso terapêutico, e o faz de forma racionalizada, calculada, sustentado por diversas concepções. ${ }^{(20)}$

\section{O acesso aos serviços de saúde}

Há uma variedade de aspectos que favorecem ou limitam o acesso aos serviços de saúde, entre eles encontram-se a disponibilidade de serviços, de recursos humanos, das ofertas organizadas, de questões burocráticas que funcionam como reguladoras do sistema, como por exemplo, o cartão 
SUS, dos fluxos estabelecidos dentro do sistema, que ora funcionam como uma barreira, ora como a chave para o atendimento desejado ou necessário. Nesse sentido, os depoimentos a seguir ilustram a facilidade de acesso aos serviços:

Já saí de lá com a consulta marcada, a secretária já marca, e às vezes quando ela não marca, passa uns dois dias já liga, que nem agora tem consulta com Dr. A. para dois de março, daqui poucos dias, às nove e meia da manhã, foi ela que ligou falando. (E4)

[...] como eu tenho os documentos tudo certo, vim fazer as consultas aqui, cheguei às sete horas por aí, tinha consulta já marcada daqui do outro dia que vim trazer o exame positivo. [...] tenho uma comadre aqui em Foz e dou o telefone dela e ela me avisa. (E5)

[...] Uma semana, vim agendar aqui, porque eles mandam agendar aqui no posto, uma semana depois já fiz (se referindo ao exame de ultrassom). (E11)

O acesso deve ser universal e igualitário às ações e serviços para a promoção, proteção e recuperação da saúde, em todos os níveis de complexidade, sendo um direito fundamental a todo cidadão brasileiro.

(4) Garantir e proporcionar esse acesso tem sido um dos grandes desafios, uma vez que este atributo da atenção primária à saúde é uma dimensão do desempenho dos sistemas de saúde associada à oferta. ${ }^{(21)}$

$\mathrm{O}$ acesso envolve a consecução do cuidado a partir das necessidades e está vinculado com a resolutividade, extrapolando a simples dimensão geográfica e incluindo outros aspectos de ordem econômica, cultural, organizacional e de oferta de serviços. ${ }^{(22)} \mathrm{O}$ que se espera, portanto, é que os serviços no primeiro nível de atenção, sejam acessíveis e resolutivos na atenção às necessidades de saúde trazidas pela população.
Contudo, em relação à oferta de serviços para resolução dos problemas de saúde, têm-se aspectos dificultadores, os quais se evidenciam nos depoimentos: "[...] única coisa que está enroscando é o meu cartão do SUS, que diz que está vencido. (E1) [...] um ano para voltar no especialista, quando me chamaram, o exame não valia mais, tinha que fazer de novo, nem fui mais atrás". (E9)

A exemplo do evidenciado nos depoimentos, entre as barreiras para o acesso, têm-se aspectos políticos, econômicos, sociais, organizativos, técnicos e simbólicos, no estabelecimento de caminhos para a universalização da atenção no SUS. ${ }^{(23)}$

Considerando o cenário da tríplice fronteira, o acesso pode ser dificultado sob o prisma organizativo, pois esta é uma população que utiliza os serviços de saúde, porém, não aparece nas estatísticas para, por exemplo, que a gestão possa pleitear aporte financeiro para o atendimento, tendo como consequência direta, a dificuldade na organização da assistência dos usuários, residentes e não residentes do município. Incluindo aqui a ausência de políticas claras para o atendimento destes brasileiros, que muitas vezes, devido à desinformação, tem prejuízos diretos na assistência à sua saúde.

Cabe ainda ressaltar, sob este mesmo aspecto, que a desarticulação entre os níveis de atenção, citados no estudo, entre os níveis primário e secundário, figura como um aspecto crítico a ser enfrentado, para a ampliação do acesso aos serviços de saúde. Esta estruturação das redes de atenção a partir da atenção básica, tendo como princípio a hierarquização do SUS, é um aspecto central para a organização. ${ }^{(24)}$

As dificuldades no acesso às consultas, aos exames e atendimento especializado, com longos períodos de espera, falta de estrutura e de pessoal são falhas que contribuem para a perpetuação das iniquidades do SUS, e o impedem de seguir com excelência, os caminhos traçados por seus princípios. $^{(25)}$ 
A atenção primária à saúde como (des)coordenadora da rede de atenção

A APS é definida como o nível de um sistema de serviço de saúde que oferece a entrada no sistema para todas as novas necessidades e problemas, fornece atenção sobre a pessoa (não direcionada para a enfermidade) no decorrer do tempo, fornece atenção para todas as condições, exceto as muito incomuns ou raras, e coordena ou integra a atenção fornecida em algum outro lugar ou por terceiros. ${ }^{(21)}$ Uma das ações de coordenação da rede de atenção é o encaminhamento orientado dos usuários para os outros níveis de atenção, conforme a hierarquização do sistema de saúde local.

Os brasileiros que residem fora do país, precisam em especial desta coordenação para garantir a continuidade do seu tratamento, uma vez que além das questões específicas da saúde, alia-se a dificuldade de mobilidade e o desconhecimento de endereços e dos novos fluxos, evidenciado nos depoimentos:

[...] primeiro passei com um clínico, que não é especialista, dai que cheguei para a Dra. M. por causa da dor no pé [...] fui também no médico de estômago. [...] Dr. R. me deu encaminhamento para o médico de vista, ano passado e agora em dezembro, a assistente social me deu o papel para fazer os óculos, peguei faz uma semana, nem me acostumei com ele ainda. (E6)

Procurar o posto de saúde, primeira coisa o posto de saúde [...] Orientou a passar para outro médico do Costa Cavalcanti [...] É foi feito antes da cirurgia, foi feito um monte de exames (E10).

Percebe-se um ir e vir no interior do sistema, num tempo considerado longo, para obter o cuidado em saúde esperado. Sobre o fluxo de cuidado na rede de atenção, corroborando com os achados dessa pesquisa, estudo ${ }^{(21)}$ afirma que, com a hierarquização, a APS perde seu valor e papel como centro coordenador de toda a rede. A falta de comunicação entre seus pontos isolados, a ausência de informações pela falta de referência e contrarreferência, ausência de atenção compartilhada, conflitos entre cuidados prescritos por diversos profissionais, ausência de acompanhamento e continuidade e inexistência de registros acerca da história clínica de pacientes, acabam por gerar duplicidade de serviços, alto custo ao sistema, demora no diagnóstico e no tratamento de agravos à saúde e, até mesmo, o afastamento do usuário da APS, ficando por vezes apenas sob acompanhamento dos outros níveis de atenção. Situações estas que, devido à falta de coordenação no cuidado, refletem uma assistência pouco resolutiva e de baixa eficiência aos usuários. ${ }^{(26)}$

A atenção coordenada é justificada e necessária porque é cada vez maior o número de pessoas que sofrem de mais de uma doença e recebem cuidados de saúde de diversos profissionais e de diferentes especialidades. Uma vez que as necessidades de saúde são bastante complexas e, em geral não são adequadamente atendidas por sistemas de saúde caracterizados pela especialização e orientação profissional isolada. A inadequação pode resultar não só em necessidades não atendidas, como também em tratamentos desnecessários, duplicidade de ações e medicalização excessiva. ${ }^{(12)}$

Uma atenção primária forteé aquela que consegue oferecer aos usuários que necessitam de cuidados mais complexos, as orientações necessárias para encontrar seu caminho no sistema de saúde, através da coordenação do cuidado, ou seja, a capacidade de garantir a continuidade da atenção por meio das equipes de saúde, com o reconhecimento dos problemas que requerem seguimento constante. ${ }^{(27)}$

\section{Considerações}

Os caminhos foram percorridos com fragilidades na integralidade da atenção e na equidade da informação com relação às respostas do sistema 
de saúde em relação à pluralidade de carências dos usuários. Ainda, percebeu-se necessidade de revisão dos serviços quanto ao cumprimento dos atributos da atenção primária na atenção aos brasileiros residentes fora do país. Houve utilização similar dos subsistemas do itinerário terapêutico, entretanto o subsistema profissional foi o mais mencionado.

Quanto ao acesso e a resolutividade especificamente, percebeu-se satisfação com o atendimento e o itinerário terapêutico percorrido, embora existam limites associados ao acesso e ao fluxo de cuidado na atenção a estes usuários, relacionados tanto a fatores socioeconômicos quanto a barreiras organizacionais e geográficas. Entretanto, para aqueles que obtiveram a acessibilidade à unidade de saúde, os fatores mencionados não foram impeditivos à assistência. Contudo, compreendese o acesso como includente da acessibilidade e para além dela, como o cuidado resolutivo em saúde. Desse modo, estiveram presentes muitas deficiências no cuidado, que são as mesmas para todos os brasileiros, independentemente do local de residência.

Menciona-se como limitação do estudo o número de entrevistados, a realização das entrevistas em uma única unidade de saúde, embora esta seja o ponto de atenção de referência institucionalizada, com uma mesma população usuária dos serviços de saúde do Brasil. Sugerem-se estudos futuros ampliando a apreensão de opiniões e itinerários terapêuticos percorridos em serviços de saúde de outros pontos de atenção dos países que integram a região de fronteira, ou ainda abrangendo o acesso da população a outros níveis de atenção à saúde.

\section{Referências}

1 Ferrari M. As noções de fronteira em geografia. Rev Perspectiva Geográfica. 2014; 9(10):1-25.

2 Ministério da Integração Nacional (BR). CDIF. Comissão Permanente para o Desenvolvimento e a Integração da Faixa de Fronteira. Bases para uma proposta de desenvolvimento e integração da faixa de fronteira. Grupo de trabalho interfederativo de integração fronteiriça. [Internet] 2010. [citado 2016 ago. 11]. Disponível em: http://www.mi.gov.br/c/ document_library/get_file?uuid=ab3fdf20-dcf6-43e19e64-d6248ebd1353\&groupId=10157.

3 Azevedo ST, Souza EBC. SIS-Fronteiras: o acesso à saúde para estrangeiros nos municípios paranaenses margeados pelo lago de Itaipu. Em: Anais da $8^{a}$ Semana Acadêmica e $8^{\text {a }}$ Expedição Geográfica: ensino, práticas e formação em Geografia; 2013; Cascavel: Unioeste; 2013.

4 Ministério da Saúde(BR). Política Nacional deAtenção Básica. Portaria n ${ }^{\circ} 2.436$, de 21 de setembro de 2017. [Internet]. 2017 [citado 2017 out 11]. Disponível em: http://bvsms.saude.gov.br/bvs/saudelegis/gm/2017/ MatrizesConsolidacao/comum/250584.html.

5 Foz do Iguaçu. Secretaria Municipal da Saúde. Relatório Anual de Gestão 2015. [Internet] 2015. [citado 2017 jan 6]. Disponível em: http://www.pmfi. pr.gov.br/ArquivosDB?idMidia=97328.

6 Ferreira DC, Silva GA. Caminhos do cuidado: itinerários terapêuticos de pessoas que convivem com HIV. Cien Saude Colet. 2012; 17(11):3087-98.

7 Mattosinho MMS, Silva DMGV. Itinerário terapêutico do adolescente com diabetes mellitus tipo 1 e seus familiares. Rev Latino-Am Enferm. 2007; 15(6):1113-9.

8 Gil AC. Como elaborar projetos de pesquisa. 4a. ed. São Paulo: Atlas; 2008.

9 Minayo MCS. O desafio do conhecimento: pesquisa qualitativa em saúde. 14a. ed. São Paulo: Hucitec; 2014.

10 Albuquerque MSV, Lyra TM, Farias SF, Mendes MFM, Martelli PJL. Acessibilidade aos serviços de saúde: uma análise a partir da Atenção Básica em Pernambuco. Saúde em Debate. 2014; 38(3):182-94.

11 Garcia VM, Reis RK. Perfil de los usuarios atendidos en una unidad de emergencia. Rev. Bras. Enferm. 2014; 67(2):261-7. Doi: 10.5935/00347167.20140035 .

12 Viegas APB, Carmo RF, Luz ZMP. Fatores que influenciam o acesso aos serviços de saúde na visão de profissionais e usuários de uma unidade básica de referência. Saúde Soc. 2015; 24(1):100-112.

13 Brasil. Agência Nacional de Saúde Suplementar. Sala de situação. Dados e indicadores do setor. [Internet]. 2016. [citado 2016 ago 13]. Disponível em: http:// www.ans.gov.br/perfil-do-setor/dados-e-indicadoresdo-setor/sala-de-situacao. 
14 Fertonani HP, Pires DEP, Biff D, Scherer MDA. Modelo assistencial em saúde: conceitos e desafios para a atenção básica brasileira. Cien Saude Colet. 2015; 20(6):1869-78.

15 Pinho PA, Pereira PPG. Therapeutic itineraries: paths crossed in the search for care. Interface. 2012; 16(41):435-47.

16 Silva-Junior ND, Gonçalves G, Franklin D. Escolha do itinerário terapêutico diante dos problemas de saúde: considerações socioantropológicas. Em: Anais do $3^{\circ}$ Encontro Baiano de Estudos em Cultura; 2012; Cachoeira: Recôncavo da Bahia; 2012.

17 Seixas CT, Merhy EE, Baduy RS, Slomp-Junior H. La integralidad desde la perspectiva del cuidado en salud: una experiencia del Sistema Único de Salud en Brasil. Salud Colectiva. 2016; 12(1):113-23.

18 Kalichman AO, Ayres JRCM. Integralidade e tecnologias de atenção à saúde: uma narrativa sobre contribuições conceituais à construção do princípio da integralidade no SUS. Cad Saude Publica. 2016; 32(8): e00183415.

19 Ayres JRCM. Cuidado: trabalho, interação e saber nas práticas de saúde. Rev Baiana Enferm. 2017; 31(1):3-4.

20 Slomp-Junior H, Feuerwerker LCM, Merhy EE. Histórias de vida, homeopatia e educação permanente: construindo o cuidado compartilhado. Cien Saude Colet. 2015; 20(6):1795-803
21 Starfield B. Atenção primária: equilíbrio entre necessidades de saúde, serviços e tecnologia. Brasília: Unesco/Ministério da Saúde; 2002.

22 Finkler AL, Viera CS, Tacla MTGM, Toso BRGO. O acesso e a dificuldade na resolutividade do cuidado da criança na atenção primária à saúde. Acta Paul Enferm. 2014; 27(6):548-53.

23 Assis MMA, Jesus WLA. Acesso aos serviços de saúde: abordagens, conceitos, políticas e modelo de análise. Cien Saude Colet. 2012; 17(11): 2865-75.

24 Lima SAV, Silva MRF, Carvalho EMF, Pessoa EAC, Brito ESV, Braga JPR. Elementos que influenciam o acesso à atenção primária na perspectiva dos profissionais e dos usuários de uma rede de serviços de saúde do Recife. Physis [Internet]. 2015; 25(2):635656. [citado 2018 jan 6]. Disponível em: http://www. scielo.br/scielo.php?script=sci_arttext\&pid=S0103$73312015000200635 \& \operatorname{lng}=\mathrm{en}$.

25 Baracho M. Batalha entre o SUS formal e o real perpetuam as iniquidades em saúde. [Internet]. 2013. [citado 2016 ago 20]. Disponível em: http://dssbr. org/site/2013/02/batalha-entre-o-sus-formal-e-o-realperpetuam-as-iniquidades-em-saude.

26 Fonseca-Viegas SM, Mattos-Penna CM. A construção da integralidade no trabalho cotidiano da equipe saúde da família. Esc. Anna Nery. 2013; 17(1):133-41.

27 Mendes EV. A construção social da atenção primária à saúde. Brasília: CONASS; 2015. 